\title{
Epidemiology and Outcomes of Acute Kidney Diseases: A Comparative Analysis
}

\author{
Emily J. See ${ }^{a, b, c}$ Kevan R. Polkinghorne ${ }^{d, e, f}$ Nigel D. Toussaint ${ }^{g, h}$ \\ Michael Bailey ${ }^{\mathrm{a}, \mathrm{i}}$ David W. Johnson ${ }^{\mathrm{j}, \mathrm{k}, \mathrm{l}, \mathrm{m}}$ Rinaldo Bellomo ${ }^{\mathrm{a}, \mathrm{c}, \mathrm{n}}$
}

aSchool of Medicine, University of Melbourne, Melbourne, VIC, Australia; ${ }^{b}$ Centre for Integrated Critical Care, University of Melbourne, Melbourne, VIC, Australia; 'Department of Intensive Care, Austin Hospital, Heidelberg, VIC, Australia; dSchool of Medicine, Monash University, Melbourne, VIC, Australia; 'Department of Nephrology, Monash Health, Clayton, VIC, Australia; 'Department of Epidemiology and Preventative Medicine, Australian and New Zealand Intensive Care Research Centre, Monash University, Melbourne, VIC, Australia; ${ }^{9}$ Department of Medicine, University of Melbourne, Melbourne, VIC, Australia; ' $D$ epartment of Nephrology, The Royal Melbourne Hospital, Parkville, VIC, Australia; 'Australian and New Zealand Intensive Care Research Centre, Monash University, Melbourne, VIC, Australia; jDepartment of Nephrology, Princess Alexandra Hospital, Woolloongabba, QLD, Australia; ${ }^{k}$ Centre for Kidney Disease Research, University of Queensland, Brisbane, QLD, Australia; 'Australasian Kidney Trials Network, Brisbane, QLD, Australia; ${ }^{\mathrm{m}}$ Translational Research Institute, Brisbane, QLD, Australia; ${ }^{\mathrm{n} D a t a}$ Analytics Research and Evaluation, The University of Melbourne and Austin Hospital, Melbourne, VIC, Australia

\section{Keywords}

Acute kidney disease - Chronic kidney disease - Death .

Kidney failure $\cdot$ Major adverse kidney events

\begin{abstract}
Introduction: Acute kidney diseases and disorders (AKD) encompass acute kidney injury (AKI) and subacute or persistent alterations in kidney function that occur after an initiating event. Unlike AKI, accurate estimates of the incidence and prognosis of AKD are not available and its clinical significance is uncertain. Methods: We studied the epidemiology and long-term outcome of AKD (as defined by the KDI$\mathrm{GO}$ criteria), with or without $\mathrm{AKI}$, in a retrospective cohort of adults hospitalized at a single centre for $>24 \mathrm{~h}$ between 2012 and 2016 who had a baseline eGFR $\geq 60 \mathrm{~mL} / \mathrm{min} / 1.73$ $\mathrm{m}^{2}$ and were alive at 30 days. In patients for whom follow-up
\end{abstract}

data were available, the risks of major adverse kidney events (MAKEs), CKD, kidney failure, and death were examined by Coxand competing risk regression analyses. Results: Among 62,977 patients, 906 (1\%) had AKD with AKI and 485 (1\%) had AKD without AKI. Follow-up data were available for 36,118 patients. In this cohort, compared to no kidney disease, AKD with AKI was associated with a higher risk of MAKEs (40.25 per 100 person-years; hazard ratio [HR] 2.51, 95\% confidence interval [CI] 2.16-2.91), CKD (27.84 per 100 person-years); subhazard ratio [SHR] 3.18, 95\% Cl 2.60-3.89), kidney failure (0.56 per 100 person-years; SHR $24.84,95 \% \mathrm{CI}$ 5.93-104.03), and death (14.86 per 100 person-years; HR $1.52,95 \% \mathrm{Cl} 1.20-1.92)$. Patients who had AKD without AKI also had a higher risk of MAKEs (36.21 per 100 person-years; HR 2.26, 95\% Cl 1.89-2.70), CKD (22.94 per 100 person-years; SHR $2.69,95 \% \mathrm{Cl} 2.11-3.43)$, kidney failure (0.28 per 100 person-years; SHR $12.63,95 \%$ Cl 1.48-107.64), and death (14.86
Correspondence to:

Rinaldo Bellomo, rinaldo.bellomo@ austin.org.au 
per 100 person-years; HR 1.57, 95\% Cl 1.19-2.07). MAKEs after AKD were driven by CKD, especially in the first 3 months. Conclusions: These findings establish the burden and poor prognosis of AKD and support prioritisation of clinical initiatives and research strategies to mitigate such risk.

(c) 2021 S. Karger AG, Basel

\section{Introduction}

Acute kidney injury (AKI) represents a heterogeneous syndrome characterized by an abrupt reduction in the glomerular filtration rate. It is a common complication of acute illness and is associated with adverse sequelae in the short- and long-term [1-3]. The term "acute kidney diseases and disorders" (AKD) was proposed by the Kidney Disease Improving Global Outcomes (KDIGO) guideline in 2012 to define the clinical course after AKI for patients in whom pathophysiological processes are ongoing after 7 days and to describe subacute alterations in kidney function that do not meet the criteria for either AKI or CKD [4]. Although such classifications of kidney disease states as AKI, AKD, or CKD are made on the basis of epidemiological constructs, these operational definitions facilitate the study of phenomena that are poorly understood. Since AKD is thought to represent a period of particular vulnerability during which patients are at a high risk of transitioning to $\mathrm{CKD}$, understanding the epidemiology and natural history of AKD has been highlighted by Acute Disease Quality Initiative (ADQI) and KDIGO groups as a high research priority $[5,6]$.

Despite this, AKD has not been systematically studied, and its relationship with long-term clinical outcomes is poorly understood. Data from previous studies have been variably limited by short follow-up time $[7,8]$, non-consensus definitions of exposure [7-11], clinically enriched populations [10, 12-16], lack of adjustment for confounders $[7,9,12,14-16]$, and pooling of heterogeneous data from community and hospital settings [17]. The only published study to examine AKD without AKI included patients with unknown baseline kidney function [17]. This may have introduced bias and resulted in misclassification of CKD as AKD. No study to date has quantified the burden and trajectory of AKD in an unselected hospital cohort or compared the contribution of AKD, with or without AKI, to adverse outcomes. Therefore, we aimed to estimate the incidence of AKD in hospitalized patients and to evaluate its association with major adverse kidney events (MAKEs), CKD, kidney failure, and death, stratified by AKI status. We hypothesized that AKD would occur commonly and be associated with heightened risks of each adverse event, especially when preceded by AKI.

\section{Methods}

Study Design

We performed a retrospective cohort study of patients admitted to an academic centre in Melbourne, Australia, between January 1,2012, and December 31,2016. Ethics approval was obtained prior to commencement (LNR/18/Austin/286), and the need for informed consent was waived. All reporting was performed in accordance with the Strengthening the Reporting of Observational Studies in Epidemiology (STROBE) guidelines [18]. Patients were followed until death, loss to follow-up (i.e., the date of the last available pathology test), or until December 31, 2019.

\section{Study Population}

All adult patients ( $\geq 18$ years) who were hospitalized for $>24 \mathrm{~h}$ during the study period were included if they had an estimated glomerular filtration rate (eGFR) of at least $60 \mathrm{~mL} / \mathrm{min} / 1.73 \mathrm{~m}^{2}$ at baseline and were alive at 30 days after discharge. Patients who received chronic kidney replacement therapy (haemodialysis, peritoneal dialysis, or kidney transplantation) prior to the index hospitalization were excluded.

\section{Data Collection}

Data pertaining to baseline characteristics were obtained from hospital administrative and pathology databases. Comorbidities were identified using validated International Classification of Diseases 10th Edition (ICD-10) and Australian Classification of Health Interventions (ACHI) coding algorithms [19], and included cigarette smoking, diabetes, hypertension, dyslipidaemia, cerebrovascular disease, cardiovascular disease, peripheral vascular disease, chronic heart failure, arrhythmia, valvular heart disease, lung disease, liver disease, haematological disease, and malignancy (online suppl. Appendix 1; for all online suppl. material, see www.karger.com/doi/10.1159/000515231). Any comorbidity was considered to be absent if no representative codes were identified for that individual. Relevant admission characteristics included admission type (emergency, elective, or other), admission unit (general surgery, specialty surgery, general medicine, or specialty medicine), care type (intensive care, coronary care), length of stay, and discharge destination (home, nursing home, or other). The date of death (if applicable) was extracted from the hospital medical record. All serum creatinine and eGFR measurements performed from 12 months prior to the index admission and until the end of follow-up were extracted from the laboratory database.

Baseline serum creatinine values were estimated as the median measurement between 7 and 365 days prior to admission. For patients who did not have a serum creatinine measurement performed in this period, we used the lower of the admission serum creatinine value or the value calculated using the Modification of Diet in Renal Disease Study equation assuming a baseline eGFR of $75 \mathrm{~mL} / \mathrm{min} / 1.73 \mathrm{~m}^{2}[4,20,21]$. Baseline eGFR was computed based on age, sex, and the serum creatinine level using the CKD epidemiology collaboration equation [22]. 
Definition of $A K I$ and $A K D$ Exposure

The exposure of interest was the kidney disease phenotype, categorized as AKD with AKI, AKD without AKI, and AKI alone. No kidney disease (NKD) was the reference group. Diagnoses of AKD and AKI were determined using serum creatinine measurements according to the KDIGO criteria (online suppl. Appendix 2) [6].

The KDIGO criteria define AKI as a rise in serum creatinine of at least $26.5 \mu \mathrm{mol} / \mathrm{L}$ in $48 \mathrm{~h}$ or as a $\geq 50 \%$ increase in serum creatinine from baseline within 7 days. The KDIGO criteria define AKD as the presence of $\mathrm{AKI}, \mathrm{a}>50 \%$ increase in serum creatinine, or $\mathrm{a} \geq 35 \%$ decrease in eGFR from baseline for $<3$ months, or as a newly developed eGFR $<60 \mathrm{~mL} / \mathrm{min} / 1.73 \mathrm{~m}^{2}$ for $<3$ months. Although this definition for AKD includes AKI, for the purposes of this study, the $\mathrm{AKI}$ and non-AKI subtypes of AKD were examined independently.

\section{Clinical Outcomes}

The primary outcome was the development of a MAKE at 12 months. A MAKE was defined as the composite of de novo CKD (eGFR $<60 \mathrm{~mL} / \mathrm{min} / 1.73 \mathrm{~m}^{2}$ sustained for a minimum of 3 months), kidney failure (eGFR $<15 \mathrm{~mL} / \mathrm{min} / 1.73 \mathrm{~m}^{2}$ sustained for a minimum of 3 months or initiation of chronic kidney replacement therapy), or death [23]. The date of developing a MAKE was defined as the date of the first qualifying event (i.e., the first date on which a patient's kidney function or clinical status declined sufficiently to meet the relevant criteria, without rising above that threshold again). Secondary outcomes included CKD, kidney failure, death, eGFR trajectory, and 30-day hospital readmission.

\section{Statistical Analysis}

Baseline characteristics were expressed as frequencies $(n, \%)$, means (standard deviation), or medians (interquartile range, IQR) as dictated by data type. Between-group comparisons were performed using the $\chi^{2}$ test, analysis of variance, and Kruskal-Wallis test as appropriate. Multivariable logistic regression was used to explore factors associated with developing AKD.

Predictors of the time to a MAKE were analysed using multivariable Cox regression models. The multivariable model was constructed using all available variables that were known to be biologically plausible. Results from the multivariable models were reported as hazard ratios (HRs) and 95\% confidence intervals (95\% $\mathrm{CI}$ ) and presented as a forest plot. Adjusted survival curves and the log-rank test were used to compare MAKE-free survival between phenotypes. Pre-specified first-order interaction terms between phenotype and age, sex, and comorbidities were examined. Linearity assumptions were validated by dividing continuous data into quartiles and fitting as categorical variables. Proportional hazard assumptions were confirmed graphically.

Multivariable Fine and Gray competing risk regression analyses were used to examine the risks of developing CKD and kidney failure. This approach was taken to minimize upward bias of our estimates of the incidence of non-fatal outcomes in the presence of the competing risk of death [24]. Results were reported as subhazard ratios and $95 \% \mathrm{CI}$, and presented as forest plots and cumulative incidence function curves. Other secondary outcomes, including death, hospital readmission, and eGFR trajectory, were studied using multivariable Cox regression, logistic regression, and multilevel mixed effects linear regression analyses, respectively.

To estimate the clinical significance of our findings, restricted mean survival times for MAKEs, CKD, and death were calculated according to the methodology described by Royston and Parmar
[25]. The robustness of our findings was tested by 3 sensitivity analyses. The first used an alternative definition of new or progressive CKD (a reduction in eGFR by $\geq 25 \%$ from baseline sustained for 3 months or more). The second examined predictors of the time to a MAKE over the complete period of follow-up. The third utilized multiple imputation to impute missing outcome data. Only individuals with complete comorbidity and outcome data were included; no imputation for missing data was performed. Data were analysed using Stata/MP16.0 (College Station, TX, USA). Two-sided $p$ values $<0.001$ were considered statistically significant.

\section{Results}

Between January 1, 2012, and December 31, 2016, 62,977 patients were hospitalized for $>24 \mathrm{~h}$, were alive at 30 days, and had a baseline eGFR of at least $60 \mathrm{~mL} /$ $\mathrm{min} / 1.73 \mathrm{~m}^{2}$ (see online suppl. Fig. 1).

In total, 906 patients (1\%) had AKD with AKI, 485 patients (1\%) had AKD without AKI, and 3,921 patients (6\%) had AKI alone. The remaining 57,665 patients (92\%) had NKD.

Of patients with AKI, 3,651 patients (76\%) had stage 1 AKI, 849 patients (18\%) had stage $2 \mathrm{AKI}$, and 327 patients (7\%) had stage 3 AKI. Of patients with AKD, 1,075 (77\%) had a $35 \%$ reduction in eGFR, $704(51 \%)$ had a de novo eGFR $<60 \mathrm{~mL} / \mathrm{min} / 1.73 \mathrm{~m}^{2}$, and $560(40 \%)$ had a $50 \%$ rise in the serum creatinine level, each lasting between 7 days and 3 months.

Table 1 summarizes the baseline characteristics of the study population and the frequency of missing data. The mean patient age was 55 years, half were male, and the mean baseline eGFR was $90.9 \mathrm{~mL} / \mathrm{min} / 1.73 \mathrm{~m}^{2}$. Twothirds of admissions were emergency, and $8 \%$ of patients were cared for in a critical care area at some stage during their hospitalization. Median length of stay was 3 days; however, this varied markedly by kidney disease phenotype. Common admission diagnoses included gastrointestinal, cardiovascular, and neoplastic disorders (online suppl. Table 1).

The median numbers of available serum creatinine measurements performed in the 12 months prior to admission and during admission were 1 (IQR 1-2) and 2 (IQR 1-4), respectively. Follow-up data were available for 36,118 patients. No clinically significant differences were observed in baseline characteristics between patients for whom follow-up data were or were not available (online suppl. Table 2). The median period of follow-up was 2.6 years (IQR 0.8-4.4), and the median number of available serum creatinine measurements post-discharge was 5 (IQR 2-15). 
Table 1. Baseline characteristics of 62,997 adults hospitalized for $>24 \mathrm{~h}$ who had a baseline eGFR $\geq 60 \mathrm{~mL} / \mathrm{min} / 1.73 \mathrm{~m}{ }^{2}$ by phenotype

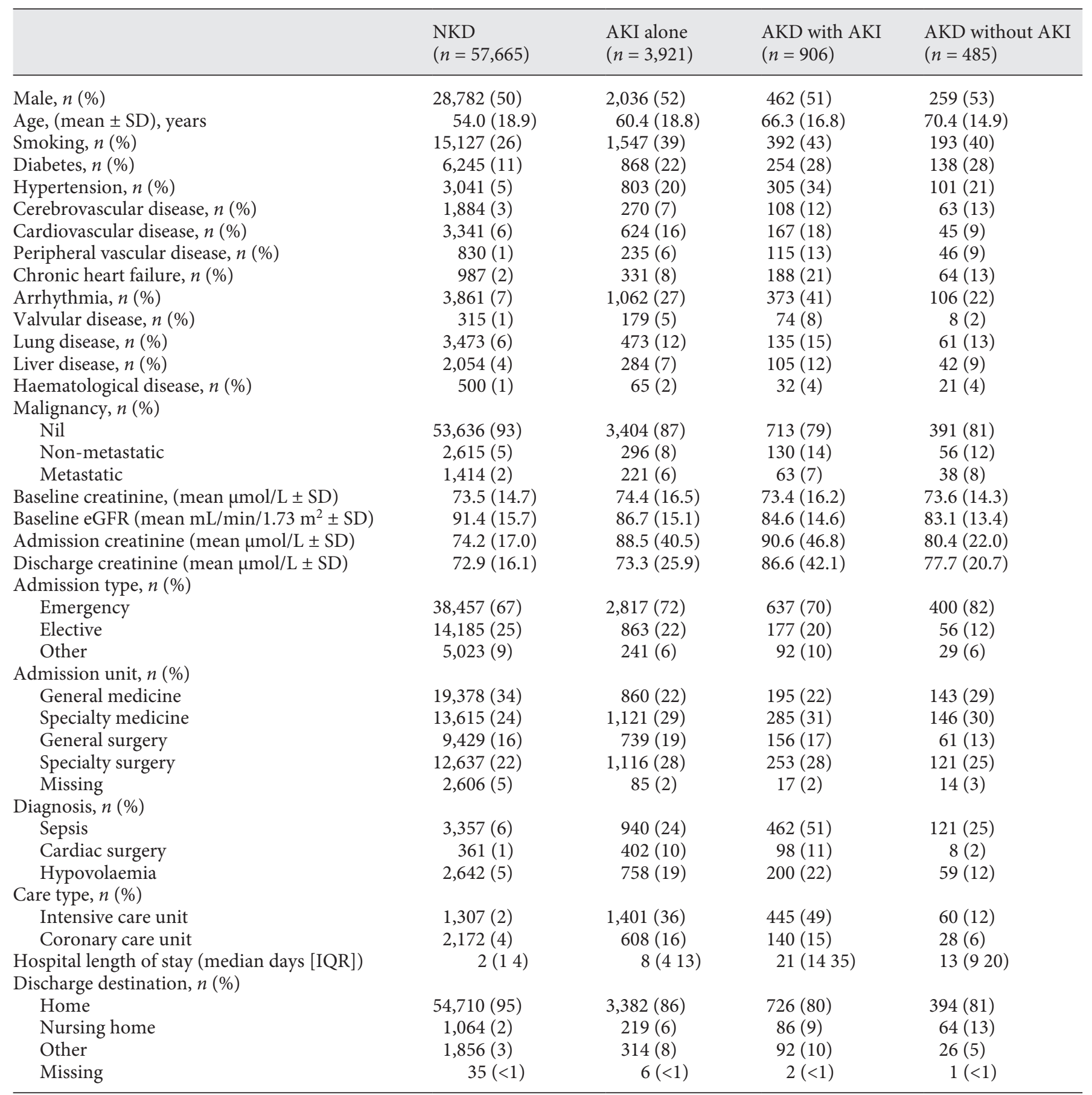

With the exception of male sex $(p=0.04)$, all other comparisons were statistically significant $(p<0.001)$. AKD, acute kidney diseases and disorders; AKI, acute kidney injury; eGFR, estimated glomerular filtration rate; IQR, interquartile range; NKD, no kidney disease; SD, standard deviation; eGFR, estimated glomerular filtration rate. 
Table 2. Overall and 12-month outcomes of 36,118 hospitalized adults with a baseline eGFR $\geq 60 \mathrm{~mL} / \mathrm{min} / 1.73 \mathrm{~m}{ }^{2}$ and at least one eGFR measurement following discharge by phenotype

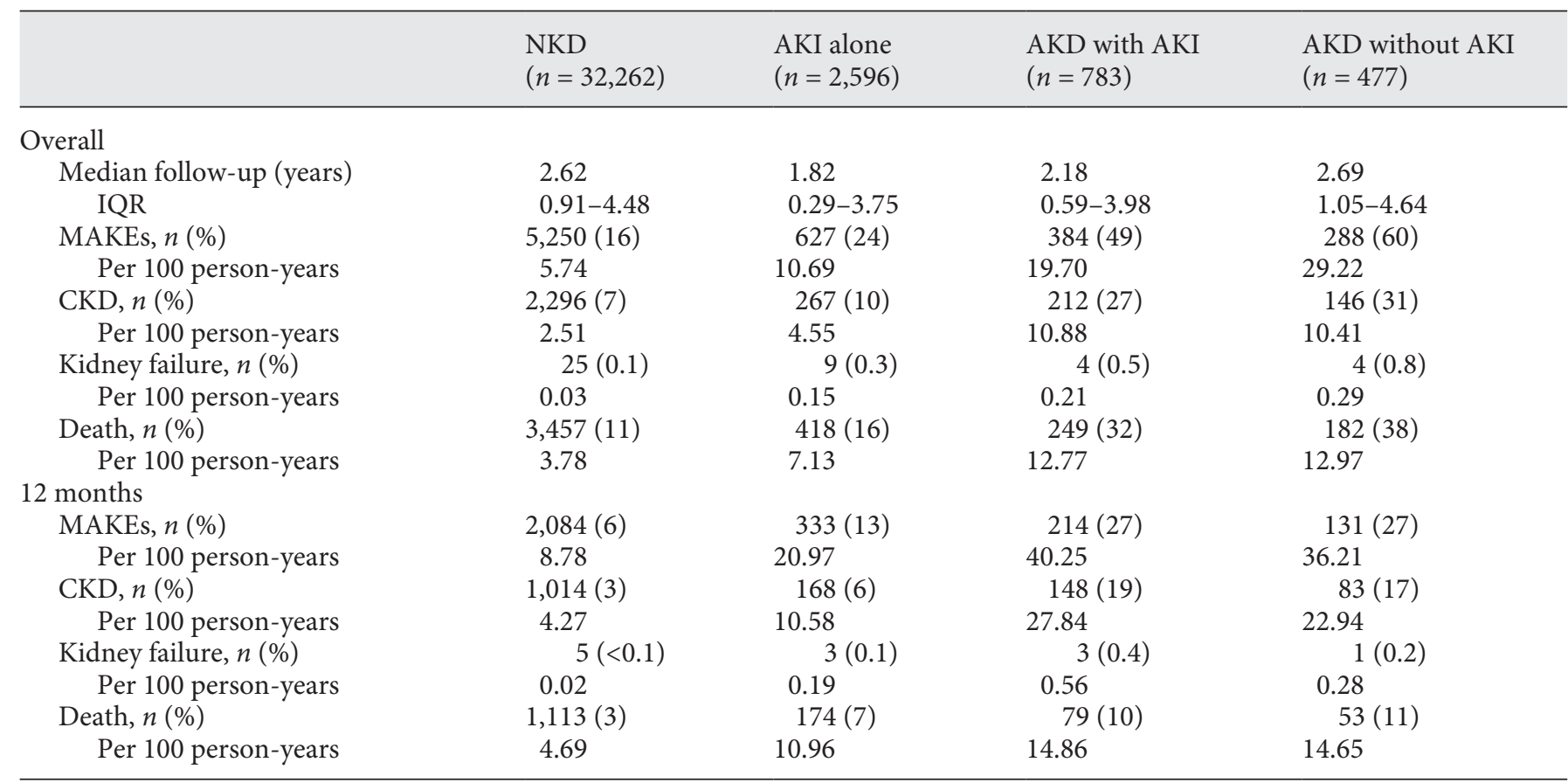

All comparisons were statistically significant $(p<0.001)$. AKD, acute kidney diseases and disorders; AKI, acute kidney injury; eGFR, estimated glomerular filtration rate; IQR, interquartile range; MAKEs, major adverse kidney events; NKD, no kidney disease.

\section{Risk Factors for Acute Kidney Disease}

Compared to those without AKD, patients with AKD were more likely to be older or to have a history of smoking, diabetes, hypertension, cerebrovascular disease, peripheral vascular disease, chronic heart failure, or liver disease (online suppl. Fig. 2). They were also more likely to have a history of haematological disease or malignancy. A multivariable model including these covariates had good discrimination for the development of AKD on internal validation (area under the receiver operating characteristic curve 0.84) (online suppl. Fig. 3).

\section{Major Adverse Kidney Events}

Follow-up data were available for 36,118 patients. Of these, a total of 6,549 patients (18\%) had a MAKE during follow-up, including 2,762 patients who (8\%) had a MAKE in the first year (online suppl. Table 3). Patients who had AKD with AKI were more likely to develop a MAKE than patients with AKD without AKI, AKI alone, or NKD (Table 2; Fig. 1). Restricted mean survival time analysis estimated that, compared to patients with NKD, MAKE-free days in the first year were 66 fewer in patients with AKD with AKI $(p<0.001), 55$ fewer in patients with
AKD without AKI $(p<0.001)$, and 20 fewer in patients with AKI alone $(p<0.001)$ (online suppl. Table 4 ; online suppl. Fig. 4). No first-order interactions between kidney disease phenotype and other covariates were identified. Proportional hazard assumptions were not violated (online suppl. Fig. 5).

\section{Chronic Kidney Disease}

De novo CKD developed in 2,921 of 36,118 patients (8\%) during follow-up, including 1,413 patients (4\%) by 12 months (Fig. 2). The risk of CKD was greater in patients with $\mathrm{AKD}$ and $\mathrm{AKI}$ or $\mathrm{AKD}$ without $\mathrm{AKI}$ than in patients with AKI alone or NKD (Table 2; online suppl. Fig. 6). Within the first year, CKD-free days were 51 fewer in patients with AKD with AKI $(p<0.001), 40$ fewer in patients with AKD without AKI $(p<0.001)$, and 12 fewer in patients with AKI alone $(p<0.001)$ than in patients with NKD (online suppl. Table 4). Cumulative incidence function curves demonstrated that the first 3 months after hospital discharge were a particularly high-risk period: approximately half $(n=303,48 \%)$ of all CKD diagnoses in patients with AKD (with or without AKI) occurred within this time. 


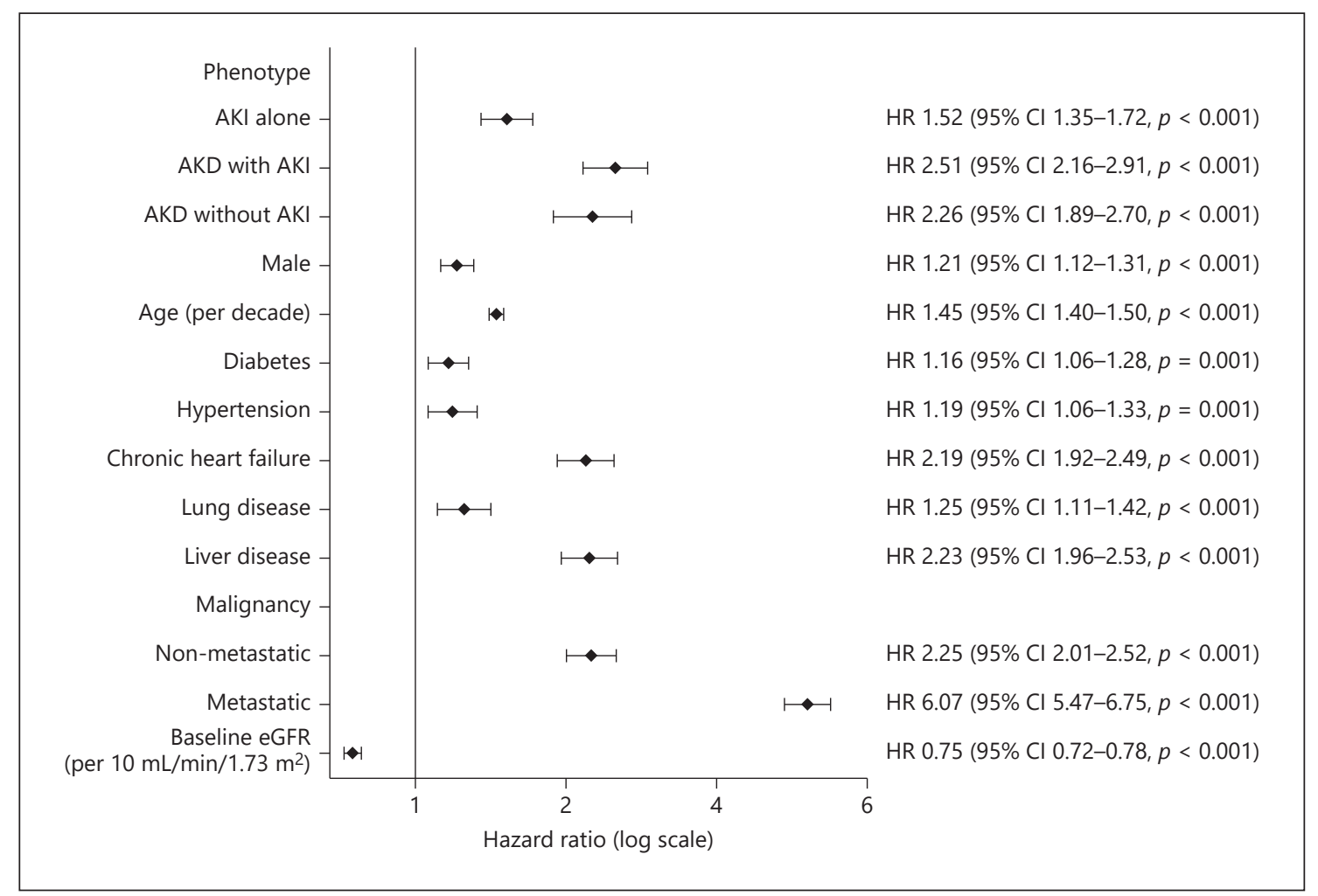

Fig. 1. Multivariable Cox regression analysis of the risk of MAKEs in 36,118 hospitalized adults with a baseline eGFR $\geq 60 \mathrm{~mL} / \mathrm{min} / 1.73 \mathrm{~m}^{2}$ and at least one eGFR measurement following discharge. MAKEs, major adverse kidney events; eGFR, estimated glomerular filtration rate; $\mathrm{AKD}$, acute kidney diseases and disorders; $\mathrm{AKI}$, acute kidney injury; HR, hazard ratio.

\section{Kidney Failure}

Kidney failure occurred in 42 of 36,118 patients $(0.1 \%)$ overall and in 12 of 36,118 patients $(0.03 \%)$ in the first year. The highest risk of kidney failure was seen in patients with AKD with AKI, followed by patients with AKD without AKI, and patients with AKI alone (Table 2; online suppl. Fig. 7). Multivariable analysis was not performed due to the small number of outcome events.

\section{Death}

Of 36,118 patients, a total of 4,306 died (12\%) during follow-up, including 1,419 patients who died in the first year (Fig. 2). The risk of death was similarly elevated in patients with $\mathrm{AKD}$ with $\mathrm{AKI}, \mathrm{AKD}$ without $\mathrm{AKI}$, and $\mathrm{AKI}$ alone, compared to patients with NKD (Table 2; online suppl. Fig. 8). Compared to patients with NKD, survival time in the first year was 16 days less in patients with AKD with AKI, 15 days less in patients with AKD without $\mathrm{AKI}$, and 8 days less in patients with AKI alone (online suppl. Table 4).

Epidemiology and Outcome of Acute Kidney Disease

\section{eGFR Trajectory}

In the 36,118 patients with available follow-up data, the median eGFR at 12 months was $89 \mathrm{~mL} / \mathrm{min} / 1.73 \mathrm{~m}^{2}$ (IQR 75-102) in patients with NKD, $86 \mathrm{~mL} / \mathrm{min} / 1.73 \mathrm{~m}^{2}$ (IQR 71-102) in patients with AKI alone, $72 \mathrm{~mL} / \mathrm{min} / 1.73$ $\mathrm{m}^{2}$ (IQR 55-91) in patients with AKD with AKI, and 70 $\mathrm{mL} / \mathrm{min} / 1.73 \mathrm{~m}^{2}$ (IQR 54-86) in patients with AKD without AKI (online suppl. Fig. 9). Compared to patients with NKD, patients with AKD with AKI had the most significant decline in eGFR, followed by AKD without AKI, and AKI alone (online suppl. Fig. 10).

\section{Hospital Readmission}

In total, 2,961 of 36,118 patients (8\%) were readmitted to hospital within 30 days. This included 2,418 patients with NKD (7\%), 339 patients with AKI alone (13\%), 127 patients with AKD with AKI (16\%), and 77 patients with AKD without AKI (16\%). The odds of hospital readmission were higher in patients with $\mathrm{AKD}$ without $\mathrm{AKI}, \mathrm{AKD}$ with AKI, and AKI alone than in patients with NKD (online suppl. Fig. 11). 


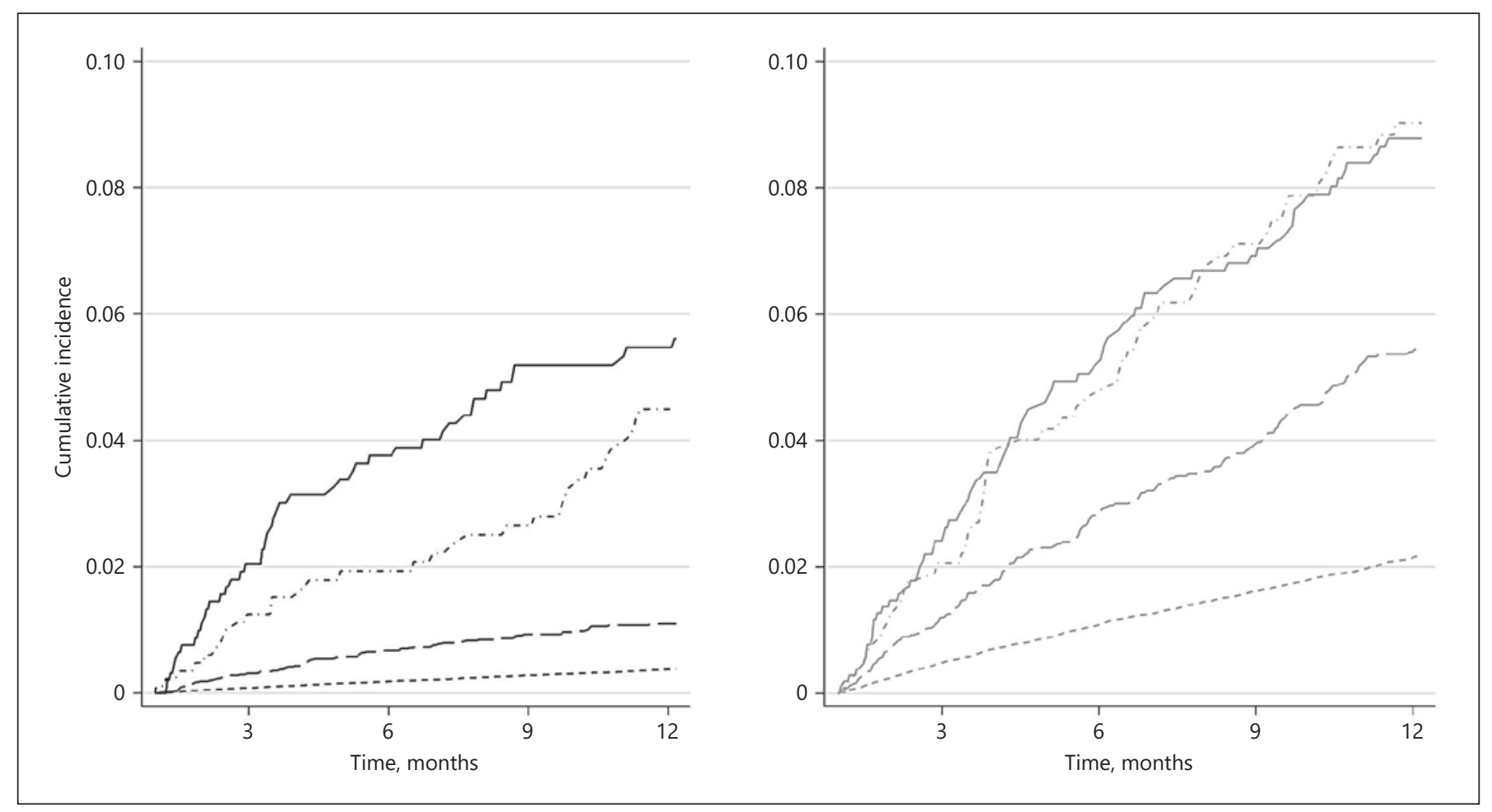

Fig. 2. Cumulative incidence of CKD (black, panel 1) and death (grey, panel 2) over the first year in 36,118 hospitalized adults with a baseline eGFR $\geq 60 \mathrm{~mL} / \mathrm{min} / 1.73 \mathrm{~m}^{2}$ and at least one eGFR measurement following discharge. Results are stratified by phenotype: NKD, short dash; AKI alone, long dash; AKD with AKI, dash dot; AKD without AKI, solid line. NKD, no kidney disease; AKD, acute kidney diseases and disorders; AKI, acute kidney injury.

\section{Sensitivity Analyses}

There were no qualitative differences in our findings when an alternative definition of CKD was used $(25 \%$ drop in eGFR from baseline sustained for 3 months) (online suppl. Table 5; online suppl. Fig. 12) when the development of a MAKE over the entire study period was examined (online suppl. Fig. 13), or when missing outcome data were imputed (online suppl. Fig. 14).

\section{Discussion/Conclusion}

\section{Key Findings}

This large study of hospitalized adults with preserved baseline kidney function demonstrates that AKD occurs in $2 \%$ and $\mathrm{AKI}$ in $6 \%$ of individuals. $\mathrm{AKD}$ is more common in patients who are older and have more comorbidities. Although patients with both AKD and AKI are an especially vulnerable group, $\mathrm{AKD}$ outcomes remain poor even in the absence of AKI. Accordingly, recognizing the development of $\mathrm{AKD}$, independent of AKI status, ap- pears to offer incremental value when estimating prognosis. De novo CKD is the predominant driver of MAKEs after AKD. Half of all CKD diagnoses in patients with AKD and/or AKI occur within the first 3 months after hospital discharge. These results fill a significant knowledge gap in the $\mathrm{AKD}$ literature and highlight important directions for future work.

\section{Relationship to Previous Studies}

A previous study by James et al. [17] found that nonAKI AKD occurred in 3.8\% of patients across hospital and community settings. Although this figure is higher than in our study, the authors did not exclude patients in whom baseline eGFR was unknown. This may have resulted in misclassification of CKD as $\mathrm{AKD}$ when an individual recorded an eGFR value $<60 \mathrm{~mL} / \mathrm{min} / 1.73 \mathrm{~m}^{2}$ for the first time. Infrequent serum creatinine testing in the community setting may also have biased their results. Nonetheless, in keeping with the findings of the present study, James et al. [17] reported that AKD without AKI was associated with increased risks of CKD, kidney failure, and death. 
Other studies have focused on AKD with AKI in clinically enriched populations and/or using non-consensus definitions of exposure. Kellum et al. [9] studied critically ill patients with stage 2 or 3 AKI and reported that $36 \%$ of their cohort went on to develop AKD. Patients with AKD had greater risks of death at 30,90 , and 365 days in their unadjusted analyses. Studies in critically ill patients with septic AKI estimated that AKD occurred in between 27 and $32 \%$ of patients, but was not associated with an increased risk of death at up to 1 year compared to patients with AKI alone [7, 13]. In cardiac populations, it has been reported that as many as $48 \%$ of patients admitted to coronary care, $47 \%$ of patients on extracorporeal membrane oxygenation, and $11 \%$ of patients undergoing cardiac surgery developed AKD $[10,12,15]$. Similar to our results, these studies found that survival between 90 days and 5 years in patients with AKD was significantly worse compared to those with NKD, as was eGFR at 2 years.

\section{Implications of Study Findings}

Our findings expand on knowledge from existing studies by providing the first robust estimate of the overall burden of AKD in hospitalized patients in a tertiary health service. Understanding the epidemiology of AKD is vital to informing clinical practice and guiding allocation of healthcare resources. AKD is not preceded by AKI in 1 in 3 patients and clinicians should be aware that these patients will not be detected by existing electronic alert algorithms for AKI. Conversely, $20 \%$ of patients with AKI will transition to $\mathrm{AKD}$, and this represents a particularly high-risk group of patients who may benefit from targeted interventions to reduce their risk of MAKEs. In the absence of specific interventions for $\mathrm{AKD}$, consideration should be given to extending clinical care bundles designed for patients with AKI. Regardless of intervention, patients with $\mathrm{AKD}$ should be followed closely for the first 3-12 months to monitor for and possibly prevent the development of de novo CKD.

\section{Strengths and Limitations}

This is the first study to report and compare the longterm outcomes of hospitalized patients with preserved baseline kidney function who develop AKD, with or without AKI. Its strengths lie in its large sample size, representative cohort, and novelty. It fills an important knowledge gap in the literature by providing insight into the incidence and long-term outcomes of patients with AKD using consensus definitions.

However, this study has several important limitations, and sources of selection bias and ascertainment bias could not be entirely excluded. Exclusion of patients lost to follow-up (i.e., those who did not undergo any post-discharge serum creatinine testing) may have introduced selection bias and led to an overestimation of the risk of MAKEs after AKD because these patients may have been at lower risk of an adverse outcome. Missing data arising from the use of non-hospital-based pathology services or other hospitals could have introduced ascertainment bias and led to an underestimation of the risk of CKD (and MAKEs) after AKD. The external validity of our findings is uncertain because the data from our single centre may not be generalizable to all health services. The limitations of using filtration markers, such as creatinine, to diagnose and assess recovery from acute and subacute kidney damage are well recognized. Although preliminary work assessing the utility of biomarkers to predict $\mathrm{AKD}$ is underway, these alternative measures of kidney function require further validation in this setting prior to clinical use $[11,15]$.

\section{Conclusions}

$\mathrm{AKD}$ is a common consequence of acute illness in hospitalized adults and is associated with adverse long-term outcomes. The future risk attributable to $\mathrm{AKD}$ is evident regardless of AKI status; its recognition as an independent clinical entity appears to offer an incremental value. Importantly, the occurrence of adverse outcomes after AKD may not be predetermined and the period immediately following injury may mark an opportunity for clinicians to mitigate further harm and optimize the chance of recovery. Future research should seek to refine our understanding of $\mathrm{AKD}$ risk factors, epidemiology, and outcomes across heterogeneous clinical settings.

\section{Acknowledgements}

We acknowledge the assistance of Ray Robbins in obtaining the dataset.

\section{Statement of Ethics}

Ethics approval was granted by the Austin Health Human Research Ethics Committee (LNR/18/Austin/286) prior to commencement, and the need for informed consent was waived because all data were de-identified. The study was conducted in line with the Declaration of Helsinki. 


\section{Conflict of Interest Statement}

D.J. has received consultancy fees, research grants, speaker's honoraria, and travel sponsorships from Baxter Healthcare and Fresenius Medical Care, consultancy fees from Astra Zeneca and AWAK, speaker's honoraria and travel sponsorships from ONO, and travel sponsorships from Amgen. All other authors have no conflicts to declare.

\section{Funding Sources}

E.S. is supported by a National Health and Medical Research Council Postgraduate Scholarship and a RACP Jacquot NHMRC Award for Excellence. D.J. is supported by a National Health and Medical Research Council Practitioner Fellowship.

\section{Author Contributions}

Research and study design: all authors; data acquisition: E.S.; data analysis/interpretation: E.S.; statistical analysis: E.S.; and supervision or mentorship: D.J., K.P., M.B., N.T., and R.B. Each author contributed important intellectual content during manuscript drafting or revision, accepts personal accountability for the author's own contributions, and agrees to ensure that questions pertaining to the accuracy or integrity of any portion of the work are appropriately investigated and resolved.

\section{References}

1 Li PK, Burdmann EA, Mehta RL. Acute kidney injury: global health alert. Minerva Urol Nefrol. 2013;65(1):1-7.

2 Silver SA, Long J, Zheng Y, Chertow GM. Cost of acute kidney injury in hospitalized patients. J Hosp Med. 2017 Feb;12(2):70-6.

3 See EJ, Jayasinghe K, Glassford N, Bailey M, Johnson DW, Polkinghorne KR, et al. Longterm risk of adverse outcomes after acute kidney injury: a systematic review and metaanalysis of cohort studies using consensus definitions of exposure. Kidney Int. 2019 Jan; 95(1):160-72.

4 Kellum J, Lameire N, Aspelin P, Barsoum RS, Burdmann EA, Goldstein SL, et al. KDIGO clinical practice guideline for acute kidney injury. Kidney Int Suppl. 2012;2(1):1-138.

5 Chawla LS, Bellomo R, Bihorac A, Goldstein SL, Siew ED, Bagshaw SM, et al. Acute kidney disease and renal recovery: consensus report of the Acute Disease Quality Initiative (ADQI) 16 Workgroup. Nat Rev Nephrol. 2017 Feb;13(4):241-57.

6 Ostermann M, Bellomo R, Burdmann EA, Doi K, Endre ZH, Goldstein SL, et al. Controversies in acute kidney injury: conclusions from a Kidney Disease: Improving Global Outcomes (KDIGO) Conference. Kidney Int. 2020 Aug;98(2):294-309.

7 Federspiel CK, Itenov TS, Mehta K, Hsu RK, Bestle MH, Liu KD. Duration of acute kidney injury in critically ill patients. Ann Intensive Care. 2018 Dec;8(1):30.

8 Legrand M, Hollinger A, Vieillard-Baron A, Dépret F, Cariou A, Deye N, et al. One-year prognosis of kidney injury at discharge from the ICU: a multicenter observational study. Crit Care Med. 2019 Dec;47(12):e953-61.

9 Kellum JA, Sileanu FE, Bihorac A, Hoste EA, Chawla LS. Recovery after acute kidney injury. Am J Respir Crit Care Med. 2017;195(6): $784-91$.
10 Matsuura R, Iwagami $\mathrm{M}$, Moriya $\mathrm{H}$, Ohtake T, Hamasaki Y, Nangaku M, et al. The clinical course of acute kidney disease after cardiac surgery: a retrospective observational study. Sci Rep. 2020 Dec;10(1):1-7.

11 Godi I, De Rosa S, Martino F, Bazzano S, Martin M, Boni E, et al. Urinary [TIMP-2] $\times$ [IGFBP7] and serum procalcitonin to predict and assess the risk for short-term outcomes in septic and non-septic critically ill patients. Ann Intensive Care. 2020 Dec;10(1):46.

12 Hsu CK, Wu IW, Chen YT, Tsai TY, Tsai FC, Fang JT, et al. Acute kidney disease stage predicts outcome of patients on extracorporeal membrane oxygenation support. PLoS One. 2020 Apr;15(4):e0231505.

13 Peerapornratana S, Priyanka P, Wang S, Smith A, Singbartl K, Palevsky PM, et al. Sepsis-associated acute kidney disease. Kidney Int Rep. 2020 Jun;5(6):839-50.

14 Patimarattananan T, Nongnuch A, Pattaranutaporn P, Unwanatham N, Jiarpinitnun C, Ngamphaiboon N. Risk and impact of delayed renal impairment in patients with locally advanced head and neck squamous cell carcinoma receiving chemoradiotherapy with cisplatin. Support Care Cancer. 2020 Jun;30:v459.

15 Chen YT, Jenq CC, Hsu CK, Yu YC, Chang $\mathrm{CH}$, Fan PC, et al. Acute kidney disease and acute kidney injury biomarkers in coronary care unit patients. BMC Nephrol. 2020 Dec; 21(1):207.

16 Xu L, Li C, Zhao L, Zhou B, Luo C, Man X, et al. Acute kidney injury after nephrectomy: a new nomogram to predict postoperative renal function. BMC Nephrol. 2020 Dec;21(1):181.

17 James MT, Levey AS, Tonelli M, Tan Z, Barry $\mathrm{R}$, Pannu N, et al. Incidence and prognosis of acute kidney diseases and disorders using an integrated approach to laboratory measurements in a universal health care system. JAMA Netw Open. 2019 Apr;2(4):e191795.
18 von Elm E, Altman DG, Egger M, Pocock SJ, Gøtzsche PC, Vandenbroucke JP. The Strengthening the Reporting of Observational Studies in Epidemiology (STROBE) statement: guidelines for reporting observational studies. J Clin Epidemiol. 2008;61(4):344-9.

19 Quan H, Sundararajan V, Halfon P, Fong A, Burnand B, Luthi JC, et al. Coding algorithms for defining comorbidities in ICD-9-CM and ICD-10 administrative data. Med Care. 2005 Nov;43(11):1130-9.

20 Závada J, Hoste E, Cartin-Ceba R, Calzavacca $\mathrm{P}$, Gajic O, Clermont G, et al. A comparison of three methods to estimate baseline creatinine for RIFLE classification. Nephrol Dial Transplant. 2010;25(12):3911-8.

21 Hoste EA, Clermont G, Kersten A, Venkataraman R, Angus DC, De Bacquer D, et al. RIFLE criteria for acute kidney injury are associated with hospital mortality in critically ill patients: a cohort analysis. Crit Care. 2006; 10(3):R73.

22 Levey AS, Stevens LA, Schmid CH, Zhang YL, Castro AF, Feldman HI, et al. A new equation to estimate glomerular filtration rate. Ann Intern Med. 2009;150(9):604-12.

23 Billings FT, Shaw AD. Clinical trial endpoints in acute kidney injury. Nephron Clin Pract. 2014;127(1-4):89-93.

24 Fine JP, Gray RJ. A proportional hazards model for the subdistribution of a competing risk. J Am Stat Assoc. 1999 Jun;94(446):496509.

25 Royston P, Parmar MK. Restricted mean survival time: an alternative to the hazard ratio for the design and analysis of randomized trials with a time-to-event outcome. BMC Med Res Methodol. 2013 Dec;13(1):152. 